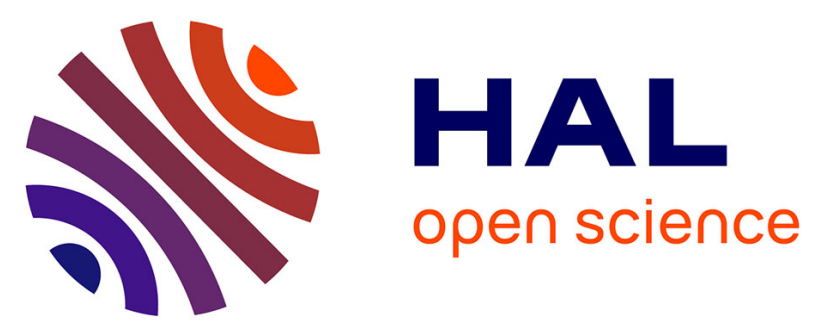

\title{
Numerical Yield Design Analysis of High-Rise Reinforced Concrete Walls in Fire Conditions
}

Jeremy Bleyer, Duc Toan Pham, Patrick de Buhan

\section{To cite this version:}

Jeremy Bleyer, Duc Toan Pham, Patrick de Buhan. Numerical Yield Design Analysis of High-Rise Reinforced Concrete Walls in Fire Conditions. Advances in Direct Methods for Materials and Structures, Springer, Cham, pp.143-161, 2017, 978-3-319-59810-9. 10.1007/978-3-319-59810-9_9 . hal-01583109

\section{HAL Id: hal-01583109 \\ https://hal-enpc.archives-ouvertes.fr/hal-01583109}

Submitted on 6 Sep 2017

HAL is a multi-disciplinary open access archive for the deposit and dissemination of scientific research documents, whether they are published or not. The documents may come from teaching and research institutions in France or abroad, or from public or private research centers.
L'archive ouverte pluridisciplinaire HAL, est destinée au dépôt et à la diffusion de documents scientifiques de niveau recherche, publiés ou non, émanant des établissements d'enseignement et de recherche français ou étrangers, des laboratoires publics ou privés. 


\title{
Numerical yield design analysis of high-rise reinforced concrete walls in fire conditions
}

\author{
Jeremy Bleyer, Duc Toan Pham and Patrick de Buhan
}

\begin{abstract}
The present contribution aims at developing a numerical procedure for predicting the failure of high rise reinforced concrete walls subjected to fire loading conditions. The stability of such structures depends, on the one hand, on thermal strains inducing a curved deformed configuration and, on the other hand, on a local degradation of the constitutive material strength properties due to the increase of temperature across the wall thickness. A three step procedure is proposed, in which the yield design (limit analysis) method is applied on two separate levels. First, an up-scaling procedure on the wall unit cell is considered as a way for assessing the generalized strength properties of the curved wall, modelled as a shell, by taking into account reduced strength capacities of the constitutive materials. Secondly, the overall stability of the wall in its fire-induced deformed configuration is assessed using lower and upper bound based on shell finite elements and the previously determined temperature-dependent strength criterion. Second-order cone programming problems are then formulated and solved using state-of-the-art solvers. Different illustrative applications are presented to investigate the sensitivity of the wall stability to geometrical parameters. Finally, the influence of imperfect connections between panels is also considered using a simple joint behaviour.
\end{abstract}

Key words: Yield design; Limit analysis; Reinforced concrete; Fire conditions; Shells; Finite Elements

Jeremy Bleyer and Patrick de Buhan

Université Paris-Est, Laboratoire Navier,

Ecole des Ponts ParisTech-IFSTTAR-CNRS (UMR 8205)

6-8 avenue Blaise Pascal, Cité Descartes, 77455 Champs-sur-Marne, FRANCE

e-mail: jeremy.bleyer@enpc.fr

Duc Toan Pham

Université Paris-Est, Centre Scientifique et Technique du Bâtiment (CSTB),

84 avenue Jean Jaurès, 77447 Marne-la-Vallée Cedex 2, FRANCE 


\section{Introduction}

Direct methods such as yield design [1] (or limit analysis in a perfectly plastic framework [2]) are now becoming increasingly popular for computing the bearing capacity of a wide variety of structures (soils [3], masonry [4], reinforced concrete $[2,5]$, etc.) thanks to the development of efficient interior point algorithms for solving the corresponding optimization problems [6].

Assessing the bearing capacity of reinforced concrete structure in fire conditions has also received increasing attention in the last decade [7, 8, 9]. The yield design approach has, for instance, recently been proposed to derive temperature-dependent interaction diagrams of reinforced concrete sections subject to a fire-induced temperature gradient [10].

The stability of high-rise reinforced concrete walls in fire condition has been investigated in [11] using yield design computations at the structure scale. The present paper is a continuation of this work and attempts at providing more insights into the failure of such structures, in particular regarding the influence of the structure geometrical configuration and the influence of imperfect connections between panels. It is a translated version of chapter 9 of the thesis [12], written in French.

\subsection{High-rise reinforced concrete panels}

Prefabricated reinforced concrete panels are increasingly used in modern high-rise industrial buildings. Such panels can be assembled either side by side in vertical strips (Figure 1-left) or stacked one over another in horizontal strips (Figure 1-right). They usually rest on concrete ground beams and can be associated to side purlins in the case of a vertical configuration or to columns in the horizontal case. The height obtained in a vertical configuration is limited by the maximal length of a panel, which typically ranges from 8 to $12 \mathrm{~m}$. On the opposite, the horizontal configuration enables to reach total heights up to $20 \mathrm{~m}$.

Such panels must usually be designed to act as fire-walls, limiting the propagation of a potential fire to other zones of the building while keeping, for a given amount of time, a sufficient mechanical strength before the complete structure collapse. Assessing the fire safety of such structures is, thus, of paramount importance and requires a more sophisticated approach than traditional design codes which are currently adapted only to panels of smaller dimensions.

\subsection{Behaviour of high-rise panels in fire conditions}

When designing traditional reinforced concrete structures in fire conditions, a reduction of stiffness and strength properties of concrete and steel as a function of temperature has to be taken into account. Including this degradation of strength properties 

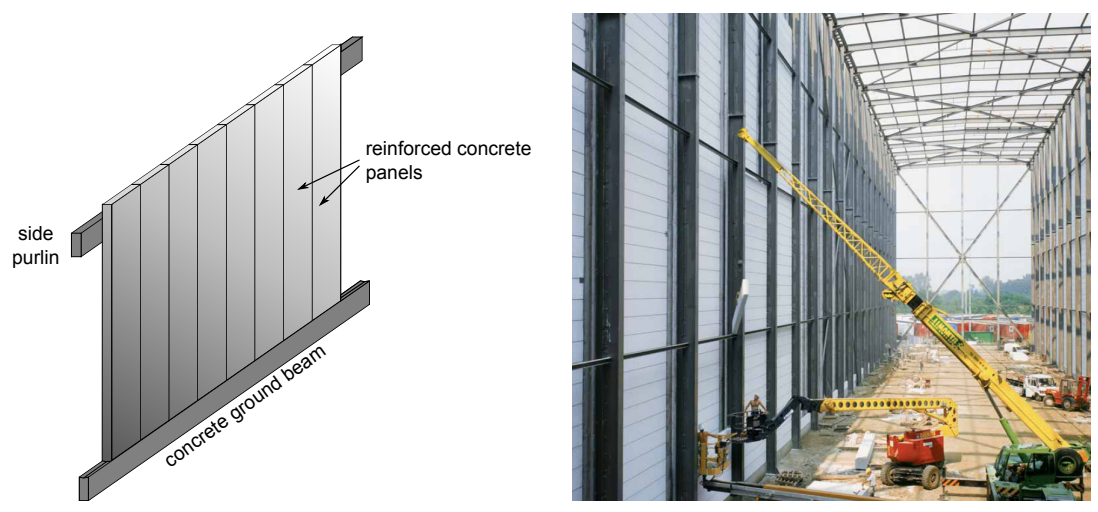

Fig. 1 High-rise reinforced concrete panels: sketch of a vertical configuration (left), high-rise industrial hall with HEBEL panels in horizontal configuration (right, source: http://www.xellahebel.fr)

for a reinforced concrete beam/plate section leads to temperature-dependent interaction diagrams in terms of membrane forces and bending moments (Figure 2-left). Nevertheless, this aspect is not sufficient to fully describe the collapse of high-rise structures.
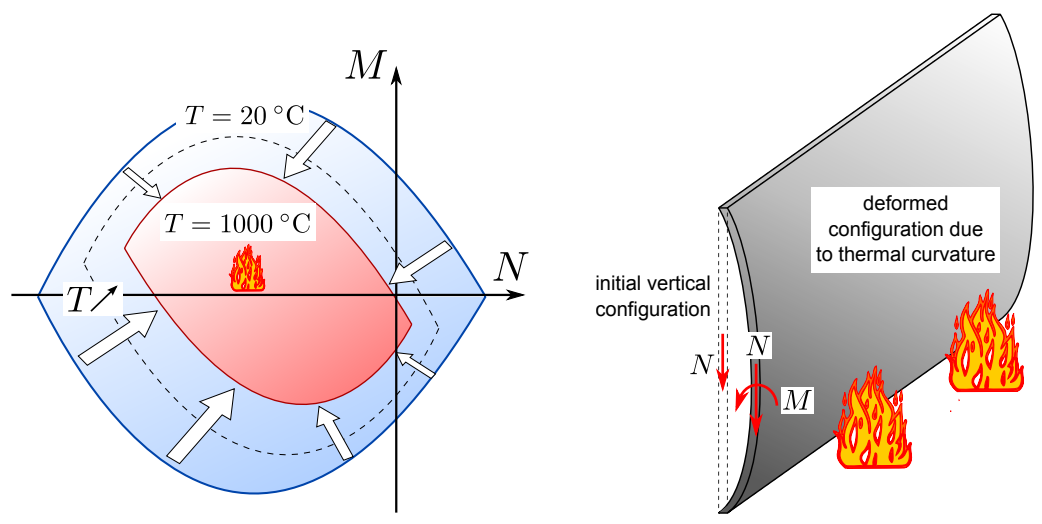

Fig. 2 Effect of fire conditions on the mechanical behaviour of high-rise panels : degradation of strength capacities (left) et geometrical changes (right)

Indeed, slender structures such as high-rise panels experience important out-ofplane displacements due to thermal deformation. Contrary to the case of smaller panels, these thermally-induced displacements can no longer be neglected and the self-weight eccentricity generates bending moments in addition to the initial compressive membrane forces (Figure 2-right). This second-order effect due to geometrical non-linearities is classically known as " $P-\Delta$-effect". Thus, it is the combined action of a degradation of material strength capacities and the effect of geometrical 
changes induced by an important temperature increase which leads to the potential collapse of the structure. Its stability analysis is, therefore, relatively complex as the geometrical configuration at which collapse will occur is not initially given but has to be computed beforehand.

\subsection{A simplified three-step procedure}

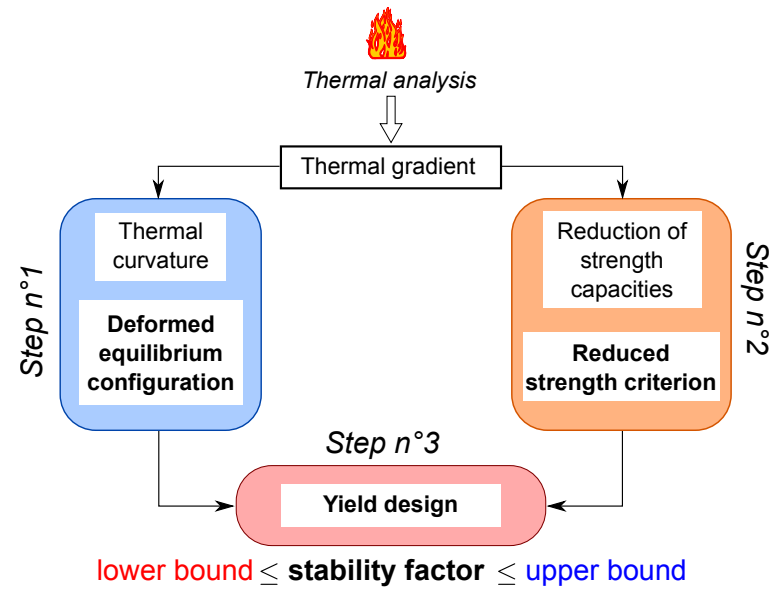

Fig. 3 A three-step procedure for assessing the fire safety of high-rise panels

In order to avoid the difficulties of a full thermo-elasto-plastic computation including geometrical non-linearities and strength properties reduction of steel and concrete, a simplified procedure is proposed and relies on three distinctive steps (Figure 3) :

- Step $\mathrm{n}^{\circ} 1$ : Determination of the deformed configuration.

Starting from a thermal gradient induced by an increase of temperature on one face of the wall, this steps amounts to compute the equilibrium configuration due to thermal deformation and self-weight.

- Step $\mathbf{n}^{\circ} 2$ : Determination of temperature-dependent strength criteria.

Starting from the same thermal gradient and in a completely independent manner, this step consists in evaluating a generalized temperature-dependent strength criterion of the reinforced concrete panel in the form of membrane-bending interaction diagrams of any wall cross-section.

- Step n 3 : Yield design analysis of the wall in its deformed configuration.

This last step consists in performing both static and kinematic approaches of yield design on the deformed configuration computed in step ${ }^{\circ} 1$ while taking 
into account the reduced strength-criterion obtained from step $n^{\circ} 2$. The outcome of this step will yield a bracketing estimate of the stability factor related to the considered configuration.

\section{Determination of the deformed configuration}

\subsection{Initial geometry}

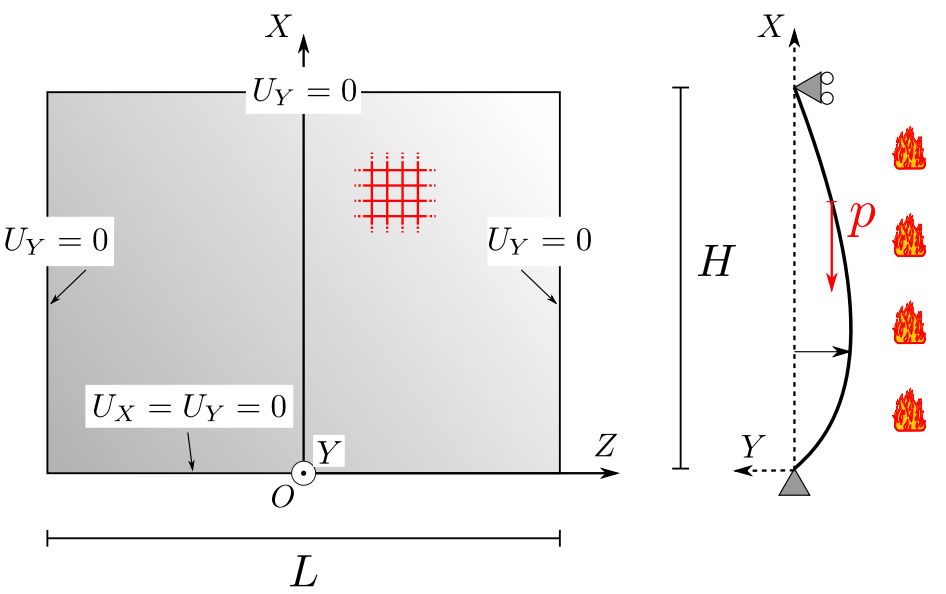

Fig. 4 Initial configuration and boundary conditions

In its initial configuration, i.e. before any thermal loading, the panel is modelled as a vertical plate of height $H$ and width $L$ in the $(O X Z)$-plane. Boundary conditions represented in Figure 4 correspond to simple supports on all edges (free rotation), while vertical displacements are fixed on the bottom side.

The panel is subjected to its own weight, represented by a uniform vertical density $p$, and to a thermal loading progressively increasing the $Y \leq 0$ face temperature from $T=20^{\circ} \mathrm{C}$ to $T=1050^{\circ} \mathrm{C}$, this corresponds to a ISO 834 fire [13] during $120 \mathrm{~min}$.

\subsection{Thermo-elastic computations}

The deformed equilibrium configuration ( $\operatorname{step} \mathrm{n}^{\circ} 1$ ) is computed using the finite element software MARC [14] according to the following points : 
- the computation is realized in the context of finite transformations : thermoelastic strains as well as rotations remain small but the change of geometry produced by horizontal out-of-plane displacements is taken into account to determine, in an iterative manner, the final equilibrium computation.

- the influence of temperature on concrete and steel elastic moduli is also taken into account using experimentally determined reduction coefficients [15].

For more details on these aspects, we refer to $[10,16]$.

\section{Determination of temperature-dependent strength criteria}

\subsection{Reduction of strength capacities}

The influence of temperature on strength properties of concrete and steel is taken into account through the adoption of (non-dimesional) reduction coefficients, denoted respectively by $k_{c}$ and $k_{y}$, the variation of which as a function of temperature is given by the Eurocode 2 norm [15] and represented in Figure 5-left. The compressive strength of concrete and the yield strength of steel at a given point of the panel characterised by a temperature $T$ will then be given by :

$$
\begin{array}{ll}
f_{c}(T)=k_{c}(T) \cdot f_{c, a m b} & \text { for concrete } \\
f_{y}(T)=k_{y}(T) \cdot f_{y, a m b} & \text { for steel }
\end{array}
$$

where $f_{c, a m b}$ and $f_{\text {y,amb }}$ represent respective strengths at ambient temperature. One can remark that the concrete compressive strength decreases gradually from ambient temperature, whereas the steel yield strength remains equal to its ambient value up to nearly $400^{\circ} \mathrm{C}$. Past this value, its strength decreases abruptly.
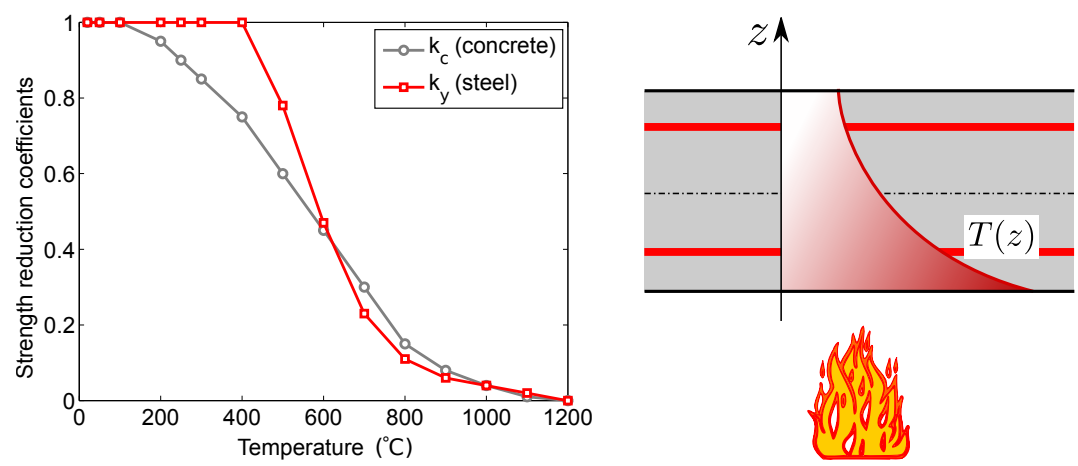

Fig. 5 Degradation of strength capacities : reduction coefficients for concrete and steel as a function of temperature (left) and thermal gradient through the panel thickness (right) 
The thermal analysis for a given fire temperature enables to compute the distribution of temperature $T(z)$ across the panel thickness (Figure 5-right). Using the previously mentioned reduction coefficients, this thermal gradient translates directly in terms of a gradient of concrete and steel strength properties across the panel thickness. For a given fire temperature, it remains now to compute the generalized strength criterion (interaction diagrams) of a plate with non-uniform strength properties.

\subsection{Generalized strength properties}

Generalizing the determination of temperature-dependent interaction diagrams for a beam [17], an up-scaling procedure is adopted to compute the generalized strength criterion of a heterogeneous plate for which an auxiliary problem is formulated on a unit cell consisting of concrete and steel rebars (Figure 6). This unit cell is represented by a parallelepiped of height $h$, the panel thickness, and of side $e$ corresponding to the spacing between steel rebars. These reinforcements are placed along four layers oriented along orthogonal directions $\underline{e}_{x}$ and $\underline{e}_{y}$ and situated at a distance $d$ from the top and bottom surfaces of the panel (now working in the local frame Oxyz).
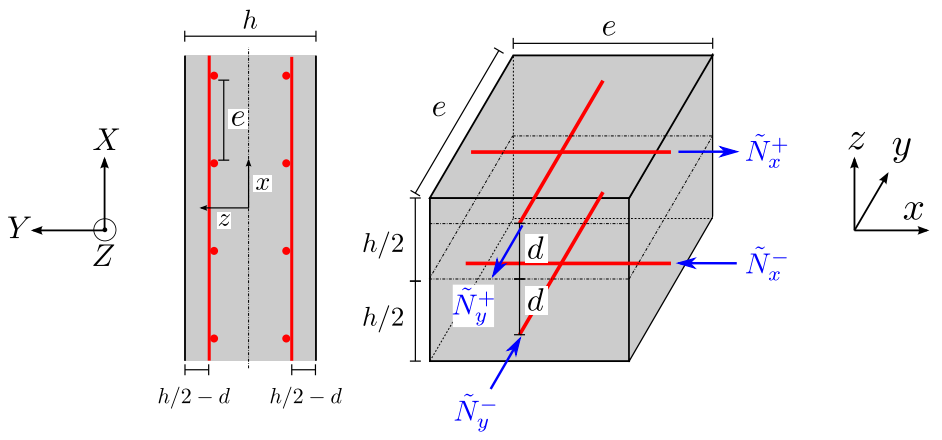

Fig. 6 Auxiliary problem used to determine the reinforced concrete panel strength criterion

The generalized strength criterion is obtained as the solution of a yield design problem with membrane forces and bending moments acting as macroscopic loading parameters, the resolution of which can be realized resorting to a 3D discretization [18].

Since we aim at modelling the deformed panel as a curved shell, we choose to take advantage of the construction of generalized strength criteria for shells developed in [19]. Indeed, if steel rebars were absent, we would have been in presence of a shell with strength properties homogeneous in its own local plane but heterogeneous across its thickness. Knowing the concrete plane stress strength criterion at all point 
across the thickness, it is possible to obtain a semi-analytical expression of the shell strength criterion, which is particularly suited for a numerical implementation [19].

The presence of steel bars is taken into account by adopting a uniaxial traction/compression modelization, embedded in the concrete matrix with a perfect bonding. Let us recall that this choice is equivalent to a lower bound approach to the true generalized strength criterion, which coincides with the latter in the limit of small volume fraction of steel and a large contrast of strength properties between steel and concrete [20].

In the following, a Mohr-Coulomb strength criterion with tension cut-off will be adopted for concrete :

$$
\underline{\underline{\sigma}} \in \mathscr{G}(z) \Leftrightarrow\left\{\begin{array}{l}
\sigma_{x x} \sigma_{y y} \geq \sigma_{x y}^{2} \\
\left(f_{c}(z)+\sigma_{x x}\right)\left(f_{c}(z)+\sigma_{y y}\right) \geq \sigma_{x y}^{2} \\
-f_{c}(z) \leq \sigma_{x x}, \sigma_{y y} \leq 0
\end{array}\right.
$$

where $z$ is the coordinate across the thickness and $f_{c}(z)$ the local concrete compressive strength obtained from (1) for a given temperature $T(z)$ at this point. As regards steel, each bar is supposed to obey a criterion of the form :

$$
\left|\tilde{N}_{x, y}^{ \pm}\right| \leq S f_{y}(z)
$$

where $\tilde{N}_{x, y}^{ \pm}$represent axial forces inside each bar, $S=\pi \phi^{2} / 4$ its cross-section area and $f_{y}(z)$ its yield strength obtained from (2).

With the previous notations, the generalized strength criterion $G$ in terms of membrane force tensor $\underline{\underline{N}}$ and bending moment tensor $\underline{\underline{M}}$ reads :

$$
(\underline{\underline{N}}, \underline{\underline{M}}) \in G \Leftrightarrow \mid \begin{aligned}
& \exists \underline{\underline{\sigma}}(z)=\sigma_{i j}(z) \underline{e}_{i} \otimes \underline{e}_{j}, N_{i}^{ \pm} \text {and } i, j=x, y \\
& N_{i j}=\int_{-h / 2}^{h / 2} \sigma_{i j}(z) d z+\frac{\tilde{N}_{i}^{+}+\tilde{N}_{i}^{-}}{e} \underline{e}_{i} \otimes \underline{e}_{i} \\
& M_{i j}=\int_{-h / 2}^{h / 2}(-z) \sigma_{i j}(z) d \xi-\frac{h / 2-d}{e}\left(\tilde{N}_{i}^{+}-\tilde{N}_{i}^{-}\right) \underline{e}_{i} \otimes \underline{e}_{i} \\
& \text { s.t. } \underline{\underline{\sigma}}(z) \in \mathscr{G}(z) \text { and }\left|\tilde{N}_{i}^{ \pm}\right| \leq S f_{y}(z) \quad \forall z \in[-h / 2 ; h / 2]
\end{aligned}
$$

which implicitly depends on the thermal gradient through the distributions of $f_{c}(z)$ and $f_{y}(z)$ across the panel thickness.

\section{Yield design analysis of the wall in its deformed configuration}

The last step of the simplified procedure consists in implementing numerically both lower and upper bound yield design approaches on the previously determined curved configuration associated to the reduced strength criterion (5). 
As mentioned earlier, the panel in its deformed configuration will be viewed as a shell modelled by an assembly of planar facets in membrane-bending interaction as described in [19]. In particular, numerical strategies for approximating the generalized strength criterion $G$ either from the inside or from the outside are employed (respectively for the lower bound static approach and the upper bound kinematic approach) to ensure the strict bounding status of the computed critical load factor $[11,19]$. Such strategies are, moreover, particularly suited for formulating the corresponding discrete optimization problems as second-order cone programs. These optimization problems are then solved using the MoseK [21] software package which implements efficient interior point algorithms.

The SOCP formulation of the global shell yield design problem, both for the upper and lower bound approaches, follows the procedure described in $[19,11]$.

The stability analysis of the structure is then assessed by computing a bracketing of the stability factor, which is here defined as the multiplicative non-dimensional factor of the loading (here the self-weight) for which the structure will collapse according to the yield design framework.

\section{Numerical investigation of the structure stability}

In the remainder of this paper, the following parameters have been retained :

$$
h=15 \mathrm{~cm}, p=3.68 \mathrm{kN} / \mathrm{m}^{2}, E_{c, a m b}=19.2 \mathrm{GPa}, f_{c, a m b}=32 \mathrm{MPa}
$$

where $E_{c, a m b}$ corresponds to the concrete Young modulus at ambient temperature. Steel rebars consist of 2 beds of HA6 steels $(6 \mathrm{~mm}$ diameter), spaced by $10 \mathrm{~cm}$, located $3 \mathrm{~cm}$ away from the bottom and top surfaces of the panel and oriented along the $\underline{e}_{X}$ and $\underline{e}_{Z}$ directions, i.e. :

$$
e=10 \mathrm{~cm}, d=4.5 \mathrm{~cm}, \phi=6 \mathrm{~mm}, f_{y, a m b}=500 \mathrm{MPa}
$$

\subsection{Influence of panel width and temperature increase}

For this first series of computations, three different geometrical configurations have been considered (Figure 7), corresponding to a slender panel in the vertical direction ( $L=3 \mathrm{~m}, H=12 \mathrm{~m})$, a square panel $(L=H=12 \mathrm{~m})$ and a slender panel in the horizontal direction $(L=30 \mathrm{~m}, H=12 \mathrm{~m})$. 
a)

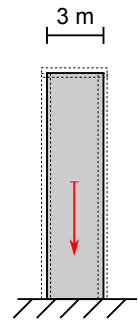

b)

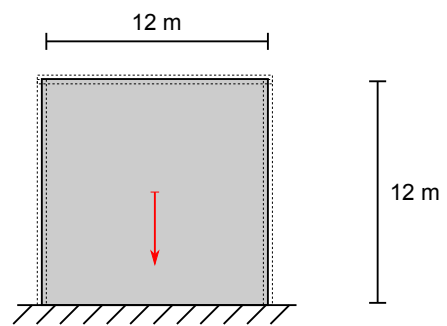

c)

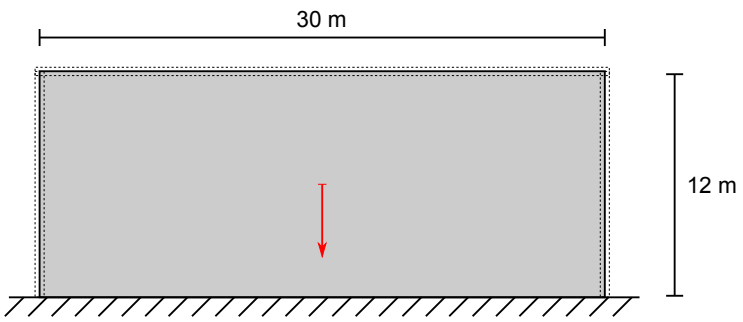

Fig. 7 Three geometrical configurations for panels with the same height $(H=12 \mathrm{~m})$ and different widths $(L=3,12,30 \mathrm{~m})$

\subsubsection{Raising temperature}

The stability of the structure, as described in (Figure 3), has been analysed every 10 min for an ISO 834 fire during $120 \mathrm{~min}$. Figure 8-left represents the evolution of the temperature profile through the panel thickness every $20 \mathrm{~min}$, starting from an initial state at ambient temperature of $20^{\circ} \mathrm{C}$.
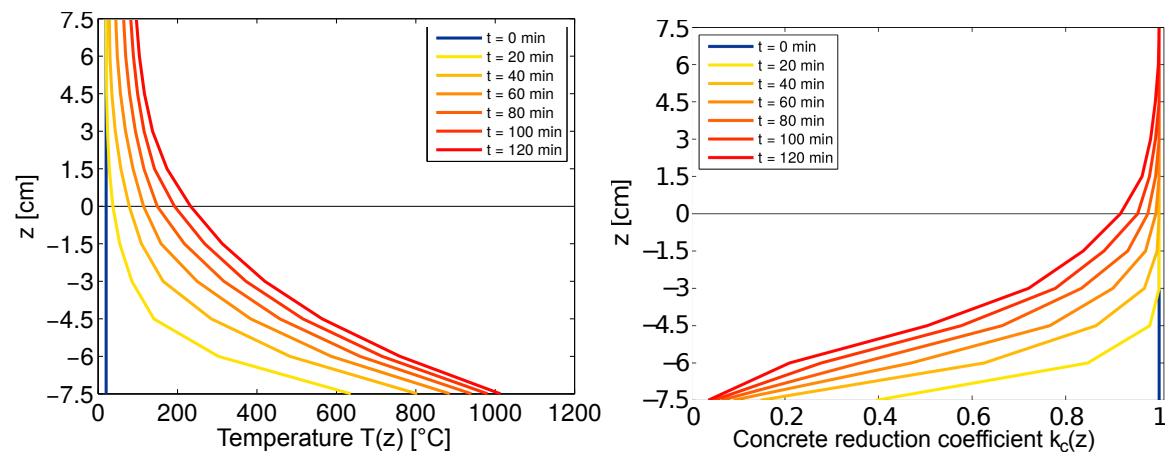

Fig. 8 Evolution of temperature profile $T(z)$ (left) and corresponding concrete reduction factor $k_{c}(z)$ (right) through the panel thickness at different times. 
It can be observed that the concrete strength loss is moderate in the upper half thickness of the panel, whereas the lower half loses in average $10 \%$ of its strength after only $20 \mathrm{~min}$ and roughly $50 \%$ after $120 \mathrm{~min}$. Finally, the bottom steel rebars start to loose their strength only after $60 \mathrm{~min}$ and is reduced by $40 \%$ after $120 \mathrm{~min}$. For the top rebars, their strength is not decreased since the temperature stays below $400^{\circ} \mathrm{C}$ at this point. All these remarks enable to interpret the evolution of the interaction diagrams during fire exposure (Figure 9). In particular, the heterogeneous distribution of strength properties across the panel thickness explains the non-symmetric shape of the diagrams in fire conditions, while its global size diminishes due to the reduction of strength properties.
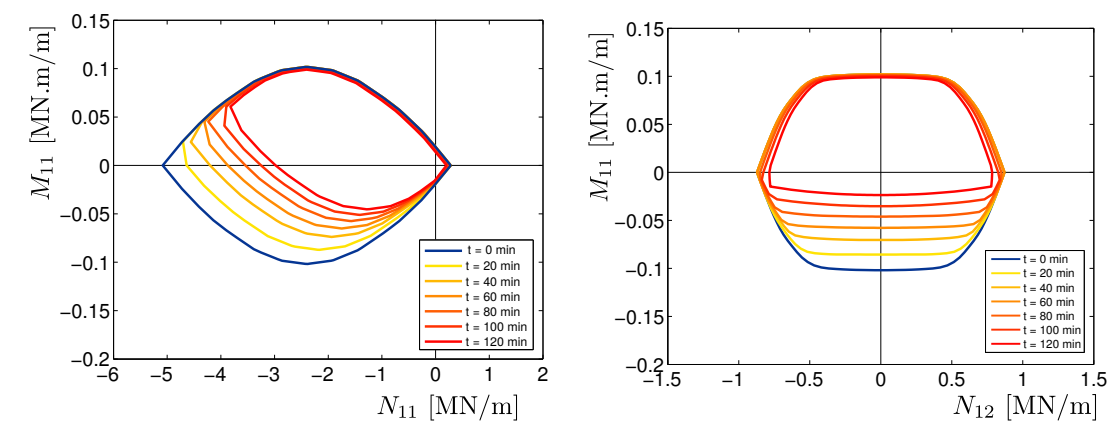

Fig. 9 Evolution of interaction diagrams at different times : left : $\left(N_{11}, M_{11}\right)$-plane, other $N_{i j}=$ $M_{i j}=0$; right : $\left(N_{12}, M_{11}\right)$-plane for $N_{11}=-2.4 \mathrm{MN} / \mathrm{m}$, other $N_{i j}=M_{i j}=0$

\subsubsection{Amplitude of geometrical changes}

In Figure 10, the maximum amplitude of the out-of-plane displacement of the thermally-induced deformed equilibrium configuration has been represented during the fire evolution and for the three panel dimensions. As expected, the width of the panel strongly influences the value of the maximum displacement. The eccentricity remains, however, moderate since the ratio between maximum out-of-plane displacement and the panel height is equal tpo $4 \%$ for $L=30 \mathrm{~m}$ and $2.5 \%$ for $L=12$ $\mathrm{m}$, justifying a posteriori the small rotation hypothesis.

\subsubsection{Stability analysis}

The resolution of the yield design problem on the deformed configuration has been realized by meshing half of the panel, using between 500 and 1000 shell elements. A value of $n=10$ (resp. $n=11$ ) has been used for the approximation of the generalized strength criterion for the static (resp. kinematic) approach (see [19]). 


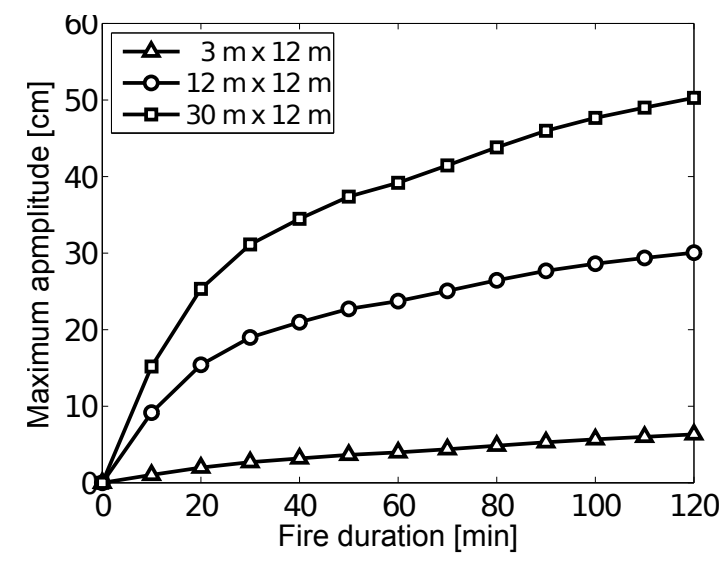

Fig. 10 Out-of-plane displacement maximum amplitude (in the $Y$ direction) of the equilibrium configuration

\begin{tabular}{|c|c|c|c|}
\hline Geometry & Static (lower bound) & Kinematic (upper bound) & Relative gap \\
\hline$L=3 \mathrm{~m}$ & 67.5 & 78.5 & $15 \%$ \\
\hline$L=12 \mathrm{~m}$ & 32.4 & 35.3 & $8.7 \%$ \\
\hline$L=30 \mathrm{~m}$ & 7.9 & 8.5 & $7.6 \%$ \\
\hline
\end{tabular}

Table 1 Bracketing of the stability factor after $120 \mathrm{~min}(H=12 \mathrm{~m})$

Table 1 collects the numerical estimates of the stability factor in terms of lower and upper bounds for the different geometries after $120 \mathrm{~min}$ of fire exposure. Again, the panel width has a strong influence on the stability factor, which is decreased by a factor 4 when the width goes from $12 \mathrm{~m}$ to $30 \mathrm{~m}$.

It is interesting to compare these values to the stability factor which would be obtained without taking into account any geometrical changes (vertical configuration) and without any degradation of strength capacities. In this case, the stability of the panel is limited by its compressive strength $N_{c, a m b}$, reached at its bottom. The associated collapse mechanism corresponds to a downwards rigid block translation (purely axial velocity discontinuity at $X=0$ in the $X \leq 0$ direction). The exact value of the stability factor is thus given by:

$$
S . F_{\text {compression }}=\frac{N_{c, a m b}}{p H} \quad \text { with } N_{c, a m b}=h f_{c, a m b}+\frac{2 S}{e} f_{y, a m b}
$$

Using the previous numerical values, we obtain here S.F. .compression $_{\text {. }}=115.14$. One can observe that this value does not depend on the panel width and that it is much larger than the values obtained when taking into account the combined effect of geometrical changes and strength capacities reduction.

Let us also remark that an analytical collapse mechanism involving 5 hinge lines considered by Pham in [10] using a plate model yielded an upper bound estimate of 
the stability factor of 36.7 for the square panel $L=H=12 \mathrm{~m}$, that is a value slightly higher than the one obtained here with the numerical upper bound approach.

As regards the $3 \mathrm{~m}$ wide panel, the optimal collapse mechanism is similar to the vertical rigid block translation previously mentioned, the collapse is thus essentially related to the compressive strength. It is possible to generalize the upper-bound estimate (8) by taking into account the degradation of the compressive strength with temperature as follows :

$$
\begin{gathered}
S . F_{\text {.compression }, T}=\frac{N_{c}(T)}{p H} \\
\text { with } N_{c}(T)=\left(\int_{-h / 2}^{h / 2} k_{c}(z) d z\right) f_{c, a m b}+\frac{S}{e}\left(k_{y}(d)+k_{y}(-d)\right) f_{y, a m b}
\end{gathered}
$$

In this case, an upper bound of S.F.compression, $T=88.1$ after $120 \mathrm{~min}$ of fire exposure is obtained, i.e. a value close to the numerical upper bound estimate for $L=3 \mathrm{~m}$.

On the other hand, the collapse mechanisms for $L=12 \mathrm{~m}$ (Figure 11-left) and $L=30 \mathrm{~m}$ (Figure 11-right) are more complex and seem to involve a bending collapse of the central part of the panel at a height of roughly $\frac{1}{4}$ to $\frac{1}{3}$ of the total height, the upper part being subjected to a rotation about $Z$ as well as a downwards vertical movement.
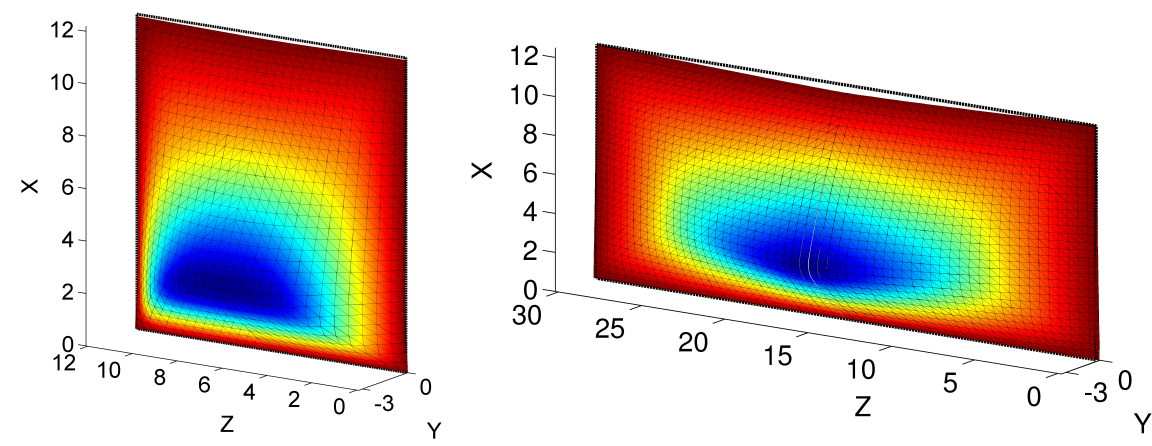

Fig. 11 Collapse mechanism for the $12 \mathrm{~m} \times 12 \mathrm{~m}$ (left) and $30 \mathrm{~m} \times 12 \mathrm{~m}$ (right) panels after 120 min (isocontours $=$ relative amplitude of the out-of-plane virtual velocity field $U_{Y}$ ).

Finally, the evolution of the stability factor for all configurations during fire exposure has also been reported in Figure 12. As a comparison, the upper bound estimate (9) corresponding to a pure compression collapse mechanism with reduced strength capacities has been represented as the black dashed line. As mentioned earlier, the stability factor for the $3 \mathrm{~m}$ wide panel is relatively close to this value.

Whereas all stability factors initially correspond to the ambient compressive strength, the wider the panel, the stronger the drop of the stability factor after a few minutes. 
On the other hand, after 40-60 min of fire, the relative decrease of the stability factor occurs at roughly the same speed for all panel width, roughly 5\% every $10 \mathrm{~min}$.

Let us also remark that the relative contribution of steel rebars to the stability factor also strongly changes with time. It is about $6 \%$ at $t=0$ min and reaches $40 \%$ for $L=12 \mathrm{~m}$ and almost $85 \%$ for $L=30 \mathrm{~m}$ after $120 \mathrm{~min}$. Steel rebars thus play an important role in limiting the strength loss of the panel during fire exposure.

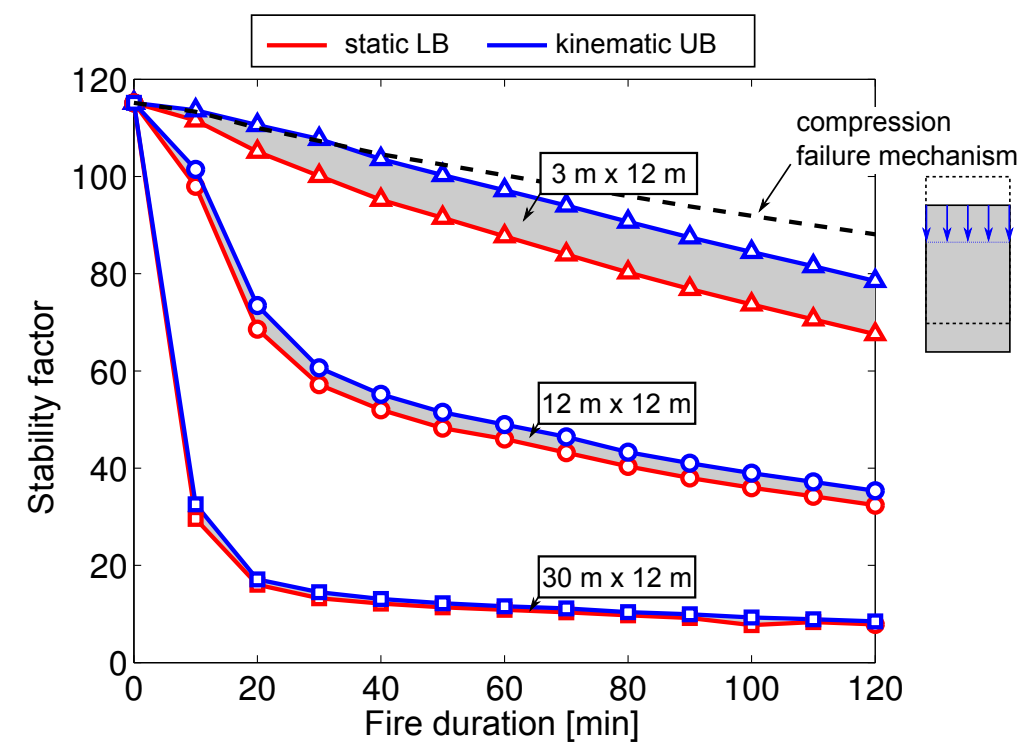

Fig. 12 Stability factor estimates evolution during fire exposure for all geometries

\subsection{Influence of panel height}

In this second series of computations, the width of the panel is now fixed to $L=12 \mathrm{~m}$ and we investigate four different values of the total height : $H=$ $4.8 \mathrm{~m}, 9.6 \mathrm{~m}, 14.4 \mathrm{~m}$, et $19.2 \mathrm{~m}$. This choice corresponds to the vertical stacking of 2, 4, 6 and 8 individual panels of dimensions $2.4 \mathrm{~m} \times 12 \mathrm{~m}$ in horizontal configuration (Figure 13).

As before, the height $H$ has an important influence on the deformed configuration amplitude. The profile of this configuration in the middle plane $(Z=6 \mathrm{~m})$ after 120 min has been represented in Figure 14-left.

The corresponding evolution of the stability factor at $120 \mathrm{~min}$ has been reported in Figure 14-right. Although an important decrease with an increase of the total 
a)

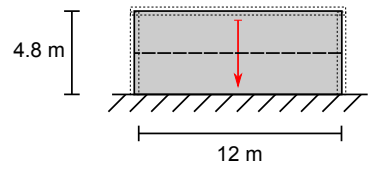

c)

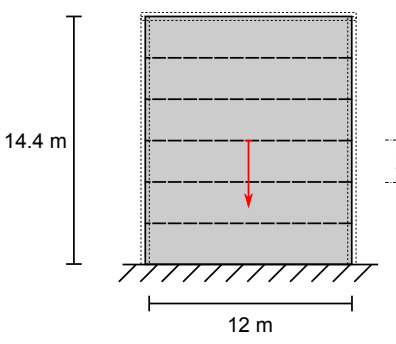

b)

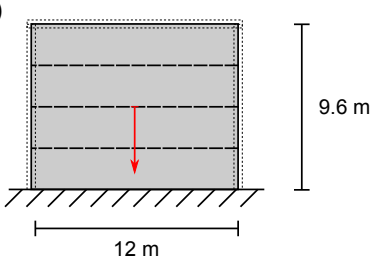

d)

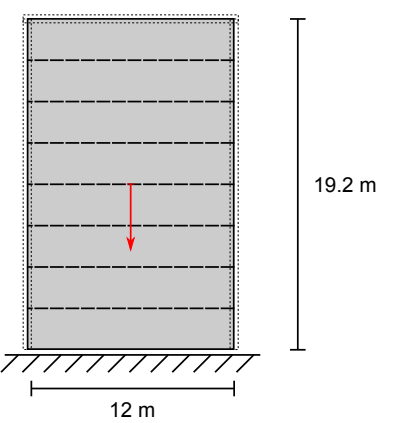

Fig. 13 For geometrical configurations of panels with the same width $(L=12 \mathrm{~m})$ stacked horizontally (total height $H=4.8,9.6,14.4,19.2 \mathrm{~m}$ )

height can be observed, its value remains around 20 for the considered numerical value. In practice, it is possible to stack such panels up to a total height of $20 \mathrm{~m}$ for modern high-rise industrial buildings. For this particular case, the stability of this kind of structure in deformed configuration is thus ensured.
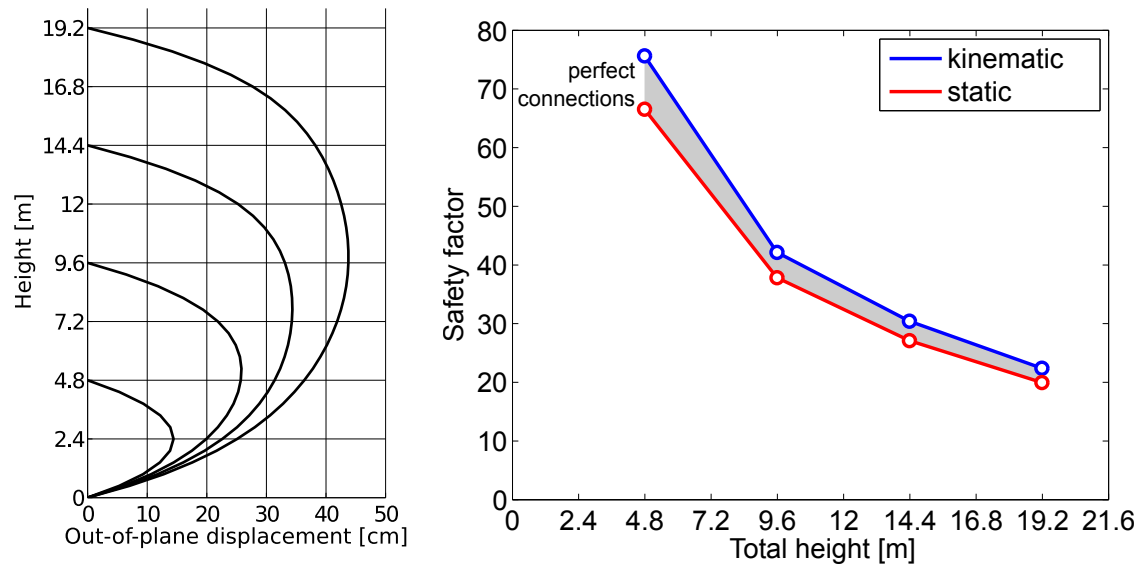

Fig. 14 Influence of total height on the panel stability : profile of the deformed configuration in the middle plane $Z=6 \mathrm{~m}$ (left) and evolution of the stability factor estimates (right) 


\subsection{Taking into account imperfect connections}

In the preceding sections, the wall has been modelled as a continuous shell with the same generalized criterion at each point of the structure. However, the connection between two individual panels may not be perfect, the side of the panels being in general assembled using male-female notches (Figure 15-left). In order to take into account the imperfect aspect of the connection, it has been chosen to model it as a joint oriented in the horizontal direction $\underline{t}$, with a vertical normal vector $\underline{n}$ in the panels plane, which offers a zero strength in bending around the joint (hinge) as well

as in tangential shear (perfect sliding). The strength criterion of such a joint can thus be written as :

$$
(\mathrm{N}, \mathrm{M}) \in \mathrm{G} \Longleftrightarrow \mid \begin{aligned}
& \exists(\underline{\underline{N}, M}) \text { s.t. } \\
& \underline{\underline{n}} \cdot \underline{\bar{N}} \cdot \mathrm{N} \\
& \underline{\underline{\underline{N}}} \cdot \underline{\underline{n}}=0 \\
& \underline{\underline{n}} \cdot \underline{\bar{M}} \cdot \underline{\underline{n}}=0 \\
& \underline{\underline{\underline{M}}} \cdot \underline{\underline{n}}=\mathrm{M} \\
& (\underline{\underline{\underline{N}}, \underline{M}}) \in G
\end{aligned}
$$

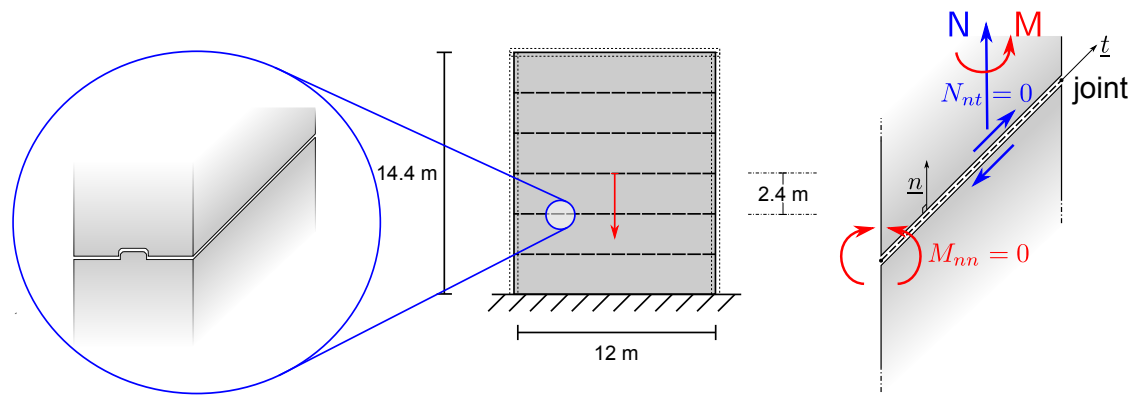

Fig. 15 Taking into account imperfect connections between panels : sketch of a possible connection (left) and mechanical model of the connection (right)

The static and kinematic approaches are then modified so as to take into account such a criterion at the connections located every $2.4 \mathrm{~m}$ in the vertical direction $X$, as represented in Figure 13. The finite element mesh is built in such a way that the edge of some elements are located along these connections, the expression of the strength criterion and the support function being modified only for these particular edges. More specifically, as regards the kinematic approach, the support function of the joint is given by :

$$
\begin{aligned}
\left.\Pi\left(\underline{n} ;\left[\llbracket u_{n}\right]\right],\left[\left[\beta_{t}\right]\right)\right) & \left.\left.=\sup _{(\mathrm{N}, \mathrm{M}) \in \mathrm{G}}\left\{\mathrm{N}\left[\llbracket u_{n}\right]\right]+\mathrm{M} \llbracket\left[\beta_{t}\right]\right]\right\} \\
& =\inf _{\widehat{v}, \widehat{\beta}} \pi\left(\underline{n} ;\left[\left[u_{n}\right] \rrbracket \underline{n}+\widehat{v} \underline{t}, \widehat{\beta} \underline{n}+\left[\llbracket \beta_{t} \rrbracket \underline{t}\right)\right.\right.
\end{aligned}
$$


where $\pi(\underline{n} ;[\underline{u}]],[[\underline{\beta}])$ is the generalized support function of the panel for velocity discontinuities.

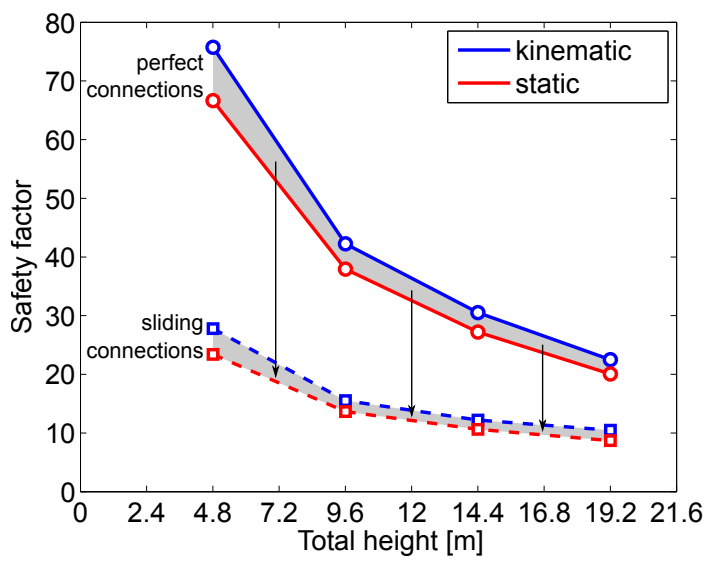

Fig. 16 Stability factor for the panel assembly by taking into account sliding connections between panels

Figure 16 represents the stability factor estimates obtained when considering imperfect connections. An important reduction of roughly $60 \%$ can be observed for the different configurations. Let us however highlight that, for the sake of simplicity, the computations have been realized on the same deformed configurations as for the perfect connections case. Now the presence of imperfect connections has certainly a significant influence on the deformed configuration amplitude, which may further reduce the stability factor.

Finally, the shape of the different collapse mechanisms with or without joints is compared in (Figures 17-20). An important difference can be observed when considering joints or not. In particular, sliding and rotation velocity discontinuities can be observed at the joints.

\section{Conclusions}

The yield design approach, along with efficient numerical tools such as interior point algorithms for conic programming, enabled to give interesting answers to the stability assessment of a complex engineering problem, involving geometrical changes as well as a reduction of strength capacities in fire conditions. Shell finite elements coupled with a specific strategy to formulate generalized strength criteria from a heterogeneous distribution of strength properties have been used to obtain satisfy- 

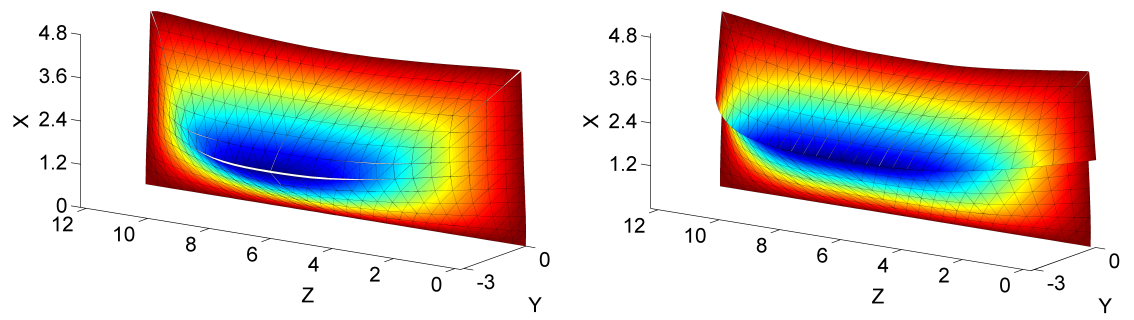

Fig. 17 Collapse mechanisms for 2 panels $(H=4.8 \mathrm{~m})$ : left: without joints $(66.6 \leq S . F . \leq 75.7)$ ; right: with joints $(23.3 \leq$ S.F. $\leq 27.7)$
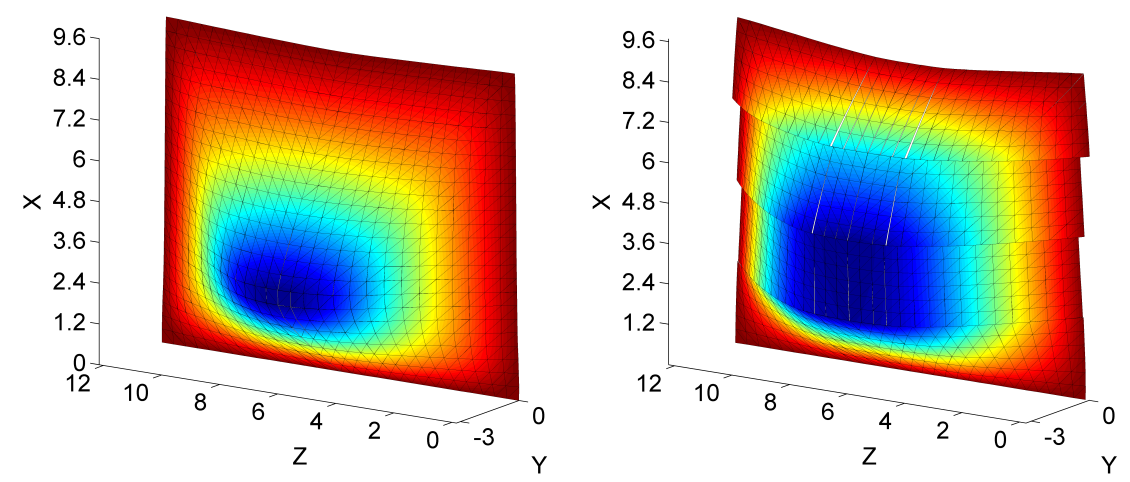

Fig. 18 Collapse mechanisms for 4 panels $(H=9.6 \mathrm{~m})$ : left: without joints $(37.8 \leq S . F . \leq 42.2)$ ; right: with joints $(13.5 \leq$ S.F. $\leq 15.4)$
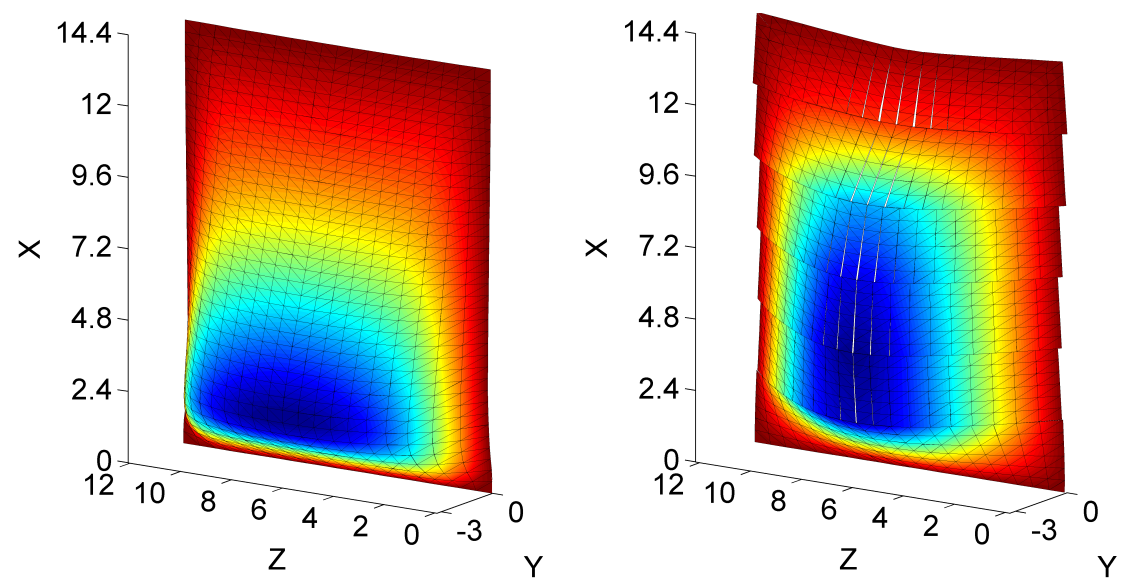

Fig. 19 Collapse mechanisms for 6 panels $(H=14.4 \mathrm{~m})$ : left: without joints $(27.1 \leq S . F$. $\leq 30.4)$ ; right: with joints $(10.5 \leq S . F . \leq 12.1)$ 

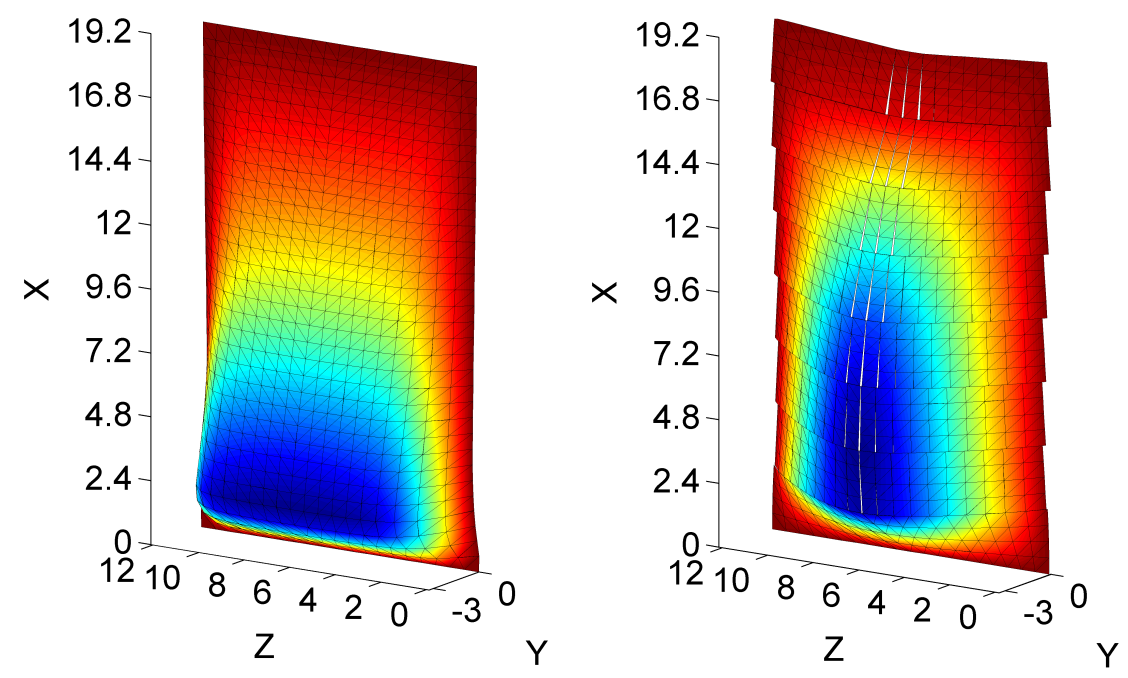

Fig. 20 Collapse mechanisms for 8 panels $(H=19.2 \mathrm{~m})$ : left: without joints $(19.9 \leq S . F . \leq 22.4)$ ; right: with joints $(8.6 \leq$ S.F. $\leq 10.4)$

ing bracketing of the stability factor (10\% to $20 \%$ relative gap) with reasonable computing times (around one minute on a standard desktop computer).

This analysis showed a great sensitivity of the stability factor with respect to the panel geometry, mainly due to its influence on geometrical changes. It has also been possible to illustrate the ability of the yield design approach to take into account imperfect connections.

Further work will be devoted to a better understanding of the link between the geometrical changes and the structure collapse. In particular, it will be interesting to investigate how yield strength properties can be accounted for in the computation of the deformed configuration without resorting to complex incremental approaches.

\section{References}

1. J. Salençon, Yield Design (London, Hoboken : ISTE Ltd., John Wiley \& Sons, Inc., 2013)

2. W.F. Chen, Plasticity in reinforced concrete (J. Ross Publishing, 2007)

3. A.V. Lyamin, S.W. Sloan, International Journal for Numerical and Analytical Methods in Geomechanics 26(2), 181 (2002)

4. G. Milani, P. Lourenço, A. Tralli, Journal of structural engineering 132(10), 1650 (2006)

5. M.P. Nielsen, L.C. Hoang, Limit analysis and concrete plasticity (CRC press, 2016)

6. K.D. Andersen, E. Christiansen, A.R. Conn, M.L. Overton, SIAM Journal on Scientific Computing 22(1), 243 (2000)

7. J.M. Franssen, J.C. Dotreppe, Fire technology 39(1), 89 (2003)

8. S. El-Fitiany, M. Youssef, Fire Safety Journal 44(5), 691 (2009)

9. R.B. Caldas, J.B.M. Sousa, R.H. Fakury, Engineering Structures 32(9), 2832 (2010) 
10. D.T. Pham, Analyse par le calcul à la rupture de la stabilité au feu des panneaux en béton armé de grandes dimensions. Thèse, Université Paris-Est (2014)

11. J. Bleyer, D.T. Pham, P. De Buhan, Proceedings of the ICE-Engineering and Computational Mechanics 168(4), 178 (2015)

12. J. Bleyer, Méthodes numériques pour le calcul à la rupture des structures de génie civil. Thèse, Université Paris-Est (2015)

13. EN 1991-1-2. Eurocode 1: Action on structures - Part 1-2: General actions - Actions on structures exposed to fire (2002)

14. MSC Software Corporation. MARC Finite Element Software. Los Angeles, CA, USA (2007)

15. EN 1992-1-2. Eurocode 2: Design of concrete structures - Part 1-2: General rules - Structural fire design (2004)

16. D.T. Pham, P. de Buhan, C. Florence, J.V. Heck, H.H. Nguyen, Engineering Structures 87, 153 (2015)

17. D.T. Pham, P. de Buhan, C. Florence, J.V. Heck, H.H. Nguyen, Engineering Structures 90, 38 (2015)

18. J. Bleyer, D.T. Pham, P. de Buhan, C. Florence, in Direct Methods for Limit and Shakedown Analysis of Structures (Springer, 2015), pp. 143-158

19. J. Bleyer, P. de Buhan, European Journal of Mechanics-A/Solids 59, 178 (2016)

20. P. de Buhan, A. Taliercio, European Journal of Mechanics - A/Solids 10(2), 129 (1991)

21. Mosek. The Mosek optimization software. Available from: http://www.mosek.com/ (December 2014). URL Available from: http://www.mosek.com/ 